\title{
Atomic Structure and Energy Distribution of Collapsed Carbon Nanotubes of Different Chiralities
}

\author{
Julia A. Baimova, ${ }^{1,2}$ Qin Fan, ${ }^{1}$ Liangcai Zeng, ${ }^{1}$ Zhigang Wang, ${ }^{1}$ Sergey V. Dmitriev, ${ }^{2,3}$ \\ Xiqiao Feng, ${ }^{4}$ and Kun Zhou ${ }^{5}$ \\ ${ }^{1}$ The Key Laboratory of Metallurgical Equipment and Control of Ministry of Education, Wuhan University of Science and Technology, \\ 947 Heping Avenue, Wuhan 430081, China \\ ${ }^{2}$ Institute for Metals Superplasticity Problems, Russian Academy of Sciences, Ufa 450001, Russia \\ ${ }^{3}$ National Research Tomsk State University, 36 Lenin Prospekt, Tomsk 634050, Russia \\ ${ }^{4}$ Department of Engineering Mechanics, Tsinghua University, Beijing 100084, China \\ ${ }^{5}$ School of Mechanical and Aerospace Engineering, Nanyang Technological University, 50 Nanyang Avenue, Singapore 639798
}

Correspondence should be addressed to Xiqiao Feng; fengxq@mail.tsinghua.edu.cn and Kun Zhou; kzhou@ntu.edu.sg

Received 10 September 2014; Accepted 17 December 2014

Academic Editor: Nay Ming Huang

Copyright (C) 2015 Julia A. Baimova et al. This is an open access article distributed under the Creative Commons Attribution License, which permits unrestricted use, distribution, and reproduction in any medium, provided the original work is properly cited.

For carbon nanotubes of sufficiently large diameter at sufficiently low temperature, due to the action of the van der Waals forces, the ground state is a bilayer graphene with closed edges, the so-called collapsed configuration. Molecular dynamics simulation of collapsed carbon nanotubes is performed. The effect of length, diameter, and chirality of the nanotubes on their properties is investigated. It is shown that collapsed nanotubes after relaxation have rippled structure which is strongly dependent on the nanotube chirality. The structural properties are studied by calculating the radial distribution function and energy distribution along various regions in the collapsed carbon nanotubes.

\section{Introduction}

Due to their extraordinary properties arising from the unique one-dimensional structure, carbon nanotubes (CNTs) have been the subject of intense theoretical and experimental studies since their discovery [1]. There have been numerous works on the mechanics of nanotubes and their properties in recent years because of their great potential for composites, sensors, electronic devices, and many other applications [26]. Calculation of the elastic properties of nanotubes confirms that they are extremely rigid in the axial direction and are most likely to distort perpendicular to the axis $[7,8]$.

Experimental investigations, as well as theoretical works ranging from full atomistic simulations to continuum modeling, have been used to study the existence of CNTs whose overall geometry differs radically from that of an ideal cylinder. Recent studies [9-17] have shown that a singlewalled CNT with an appropriate diameter has two stable states, circular and collapsed, with different potential energy.
Such an energy difference is interesting because CNTs can be used as an energy source during the transformation between the two states. The nanotubes, which are collapsed along their length, energetically are more favorable than the form with a circular cross-section [11]. Nanotube collapse was called "domino process" and modeled by molecular dynamics (MD) both at zero and finite temperatures $[9,10]$. It was also shown that collapse can significantly affect the electrical properties of CNTs [18-20].

The collapse of single-walled CNT studied via classical $\mathrm{MD}$ and molecular mechanics suggested that CNTs with a radius larger than $3 \mathrm{~nm}$ will collapse, since the energy of the collapsed state is lower than that of the circular, uncollapsed state. The energetics of the collapse of singleand multiwalled CNTs via MD was also studied in [21]. The formation of fully collapsed single-walled CNT with the atomic scale finite-element method was investigated in [22]. Interestingly, collapsed multiwalled CNTs may exist in different forms [23]. The elastic model shows that there exist 
collapsed configurations of different orders, each involving a different number of collapsed layers [24].

In particular, the production of super-strong nanotube bundles and other three-dimensional carbon structures remains a challenge to the current materials science. Since then, many researches have been carried out to understand the behavior of bundles of carbon nanotubes under high pressures [25-32].

In this paper, the MD simulation aimed at the investigation of energy and structure of collapsed single-walled CNTs is presented. Phenomena of structural transformation are shown by the radial distribution functions. Various types of rippled collapsed structures are studied.

\section{Simulation Details}

The topography of CNT is denoted by a pair of integer numbers $(n, m)$. In this study, initial atomic configurations were obtained by creating a CNT with specified parameters. Four CNTs of various chirality are considered: zigzag $(96,0)$, armchair $(55,55)$, and two chiral CNTs $(50,60)$ and $(40,70)$. The chirality indices are chosen to maintain the number of atoms in the CNTs to be almost the same at the same length and nearly the same diameter. The number of carbon atoms in the largest CNTs is about 18500. Single-walled CNTs of various lengths from $L=20$ to $200 \mathrm{~nm}$ are simulated to check the effect of length on its properties. It was shown that the length has no significant effect on some properties of collapsed structures, while it can slightly affect the other properties. The length $L=100 \mathrm{~nm}$, as the average between considered values, is chosen as the example for further descriptions.

The simulations are carried out using the large-scale atomic/molecular massively parallel simulator (LAMMPS) package [33] with the adaptive intermolecular reactive empirical bond order (AIREBO) potential [34], which has been widely used to describe the short range bonding energies among carbon atoms in many studies on the behavior and properties of graphitic polymorphs [31, 32, 35-38]. Additionally, the van der Waals forces between two carbon atoms are modeled by the Lennard-Jones potential with the equilibrium distance of $3.4 \AA$ and the bond energy of $0.024 \mathrm{eV}$. Relaxational dynamics is carried out to find the equilibrium configurations of the collapsed CNTs.

Stability of the collapsed configuration was shown in the previous studies $[9,10]$. The main goal of this work was the investigation of the collapsed state and the properties of collapsed nanotubes; that is why the initial open state of the CNT is not presented here. Collapsed nanotubes were created by application of the lateral forces applied to the atoms. After the collapsed nanotube is created, the action of the external forces is removed and the self-folded structure is relaxed to a minimum potential energy state, as shown in Figure 1(a). The relaxation of the CNT strongly depends on the chirality, which will be discussed in the sequel.

Figure 1(b) shows the schematic of a collapsed CNT. To study the energy distribution, the atoms of the self-folded CNT with its total width $H$ were divided into two groups:

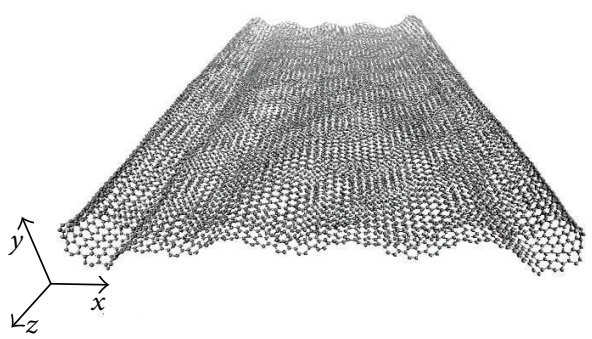

(a)

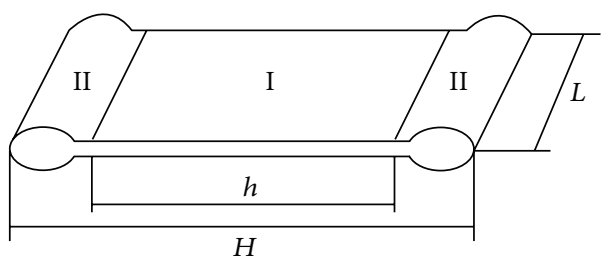

(b)

FIGURE 1: (a) Initial structure of collapsed $\mathrm{CNT}_{(50,60)}$. (b) Schematic of folded CNT. Bilayer graphene of width $h$ is the region I and the two round edges constitute the region II.

the atoms in the central flat region where one has bilayer graphene of width $h$ (region I) and the atoms in the two round edges (region II). Let $N_{1}$ denote the number of atoms in the bilayer graphene area $(h \times L)$ and $N$ the total number of atoms. Then the region II has $N_{2}=N-N_{1}$ atoms. For the collapsed structure, the opposite walls in region I are within the van der Waals distance.

The studied energies are the energy per atom of bilayer graphene, $E_{1}$ (region I), and the energy per atom for round edges, $E_{2}$ (region II). The energy in region I is

$$
E_{1}=\frac{e}{N_{1}}
$$

where $e$ is the potential energy of the area $h \times L$, and the energy in region II is

$$
E_{2}=\frac{(E-e)}{N_{2}},
$$

where $E$ is the total potential energy of the area $H \times L$.

\section{Results and Discussion}

The effect of CNT diameter $D$ on the potential energy is studied for the collapsed armchair CNTs with $L=100 \AA$. The nanotube chirality slightly affects the energies $E_{1}$ and $E_{2}$ but has no qualitative effect; that is why the results only for the armchair CNTs are presented as the example. In Figure 2, the potential energy as the function of CNT diameter is shown for $E_{1}$ (solid line) and $E_{2}$ (dashed line). It can be seen that for the small diameter the energy of collapsed edges of the nanotubes, $E_{2}$, is higher and it rises nonlinearly with decrease in $D$, which is in consistence with the previous works $[9,10]$. The competition of the van der Waals energy, providing attractive force to collapse the tube, and the elastic 


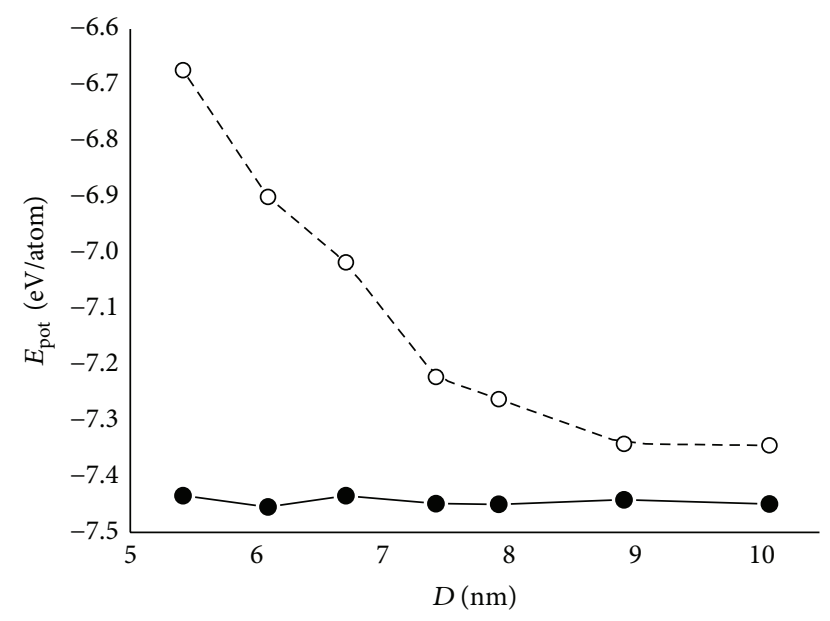

FIgURE 2: The potential energy per atom as the function of CNT diameter $D$ for region I $\left(E_{1}\right.$, solid line) and region II $\left(E_{2}\right.$, dashed line).

energy, which always tends to keep the tube circular, leads to the mechanically bistable configuration of a single-walled CNT with the two competitive energies. For tubes of smaller diameter, the elastic energy is dominant (thus the circular structure of the tube is stable), while for tubes with larger diameter the van der Waals energy is dominant, meaning that the collapsed structure of the tube is stable whereas the circular structure is metastable [7]. In the following, the properties of collapsed nanotubes are reported for the CNT diameter of $D \sim 7.5 \mathrm{~nm}$.

The effect of the CNT length on the potential energy of the CNT in the relaxed state is investigated. The behavior of chiral CNTs is quite different from that of armchair and zigzag CNTs. For the whole range of lengths, $E_{1}$ stays almost constant for all CNTs, while the energy of round edges, $E_{2}$, slowly decreases with the length increase for the armchair CNT and remains constant for other types of CNTs. The energy of chiral CNTs is found to be lower than that of the armchair and zigzag CNTs. No distinct effect of CNT length on the collapse and formation of final relaxed structures is observed since the considered CNTs have a relatively large length. The detailed analysis of the distribution of the potential energy showed that the highest energy concentrates on the ridges of the collapsed edges, while bilayer graphene (region I) has the lowest potential energy.

The relaxed states of four collapsed CNTs are shown in Figure 3 for (a) $\mathrm{CNT}_{(40,70)}$, (b) $\mathrm{CNT}_{(50,60)}$, (c) $\mathrm{CNT}_{(55,55)}$, and (d) $\mathrm{CNT}_{(96,0)}$. It can be seen that CNTs after relaxation become rippled, which is in consistence with graphene $[39,40]$. In reality, the bilayer graphene region will not remain ideally flat and its shape will depend on the spatially distributed cohesive forces due to van der Waals interactions. As it can be seen from Figure 3, a set of ripples appear in the collapsed CNTs reflecting the (in)commensurate structures in the mutual arrangements of atoms in the two graphene sheets in contact. The ripples contribute to the total potential energy of the collapsed CNTs. According to the earlier studies $[15,21]$, the most energetically favorable mutual position

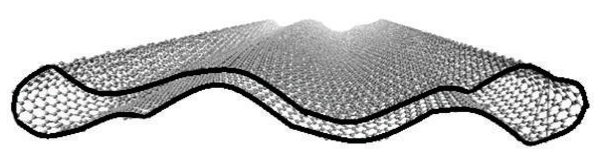

(a)

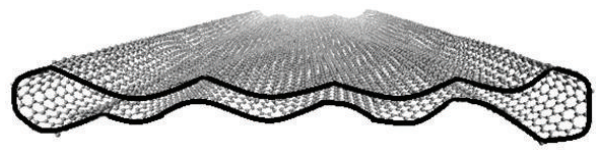

(b)

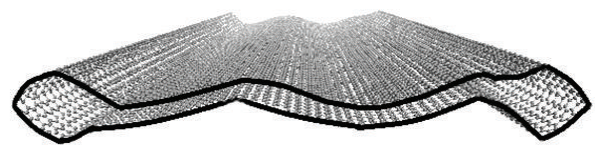

(c)

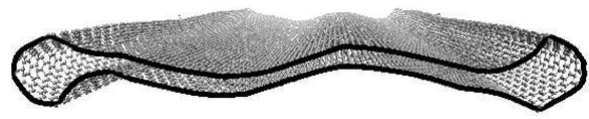

(d)

FIGURE 3: Relaxed structures of collapsed CNTs: (a) $\mathrm{CNT}_{(40,70)}$, (b) $\mathrm{CNT}_{(50,60)},(\mathrm{c}) \mathrm{CNT}_{(55,55)}$, and (d) $\mathrm{CNT}_{(96,0)}$.

of the two graphene sheets is when the $\mathrm{C}$ atoms of one sheet are above the centers of the 6-atom rings of another sheet. The ripples in bilayer graphene appear to minimize the contribution from the van der Waals bonds to the total potential energy of CNT. Only in the case of zigzag and armchair orientations the minimal energy position can be achieved by the mutual translation of the two sheets in contact. In other cases the mutual rotation of the sheets is required. In the case of collapsed CNT the mutual rotation is restricted by the closed ends of the graphene sheets. Only local atomic shifts and rotations can be realized resulting in the appearance of ripples. Not only rippling of the bilayer graphene region but also the shape of the edges is strongly dependent on the chirality of CNT. Physically, stretching of the $\mathrm{C}-\mathrm{C}$ bonds is difficult because they are stiff and strong. Therefore, during collapse, the cross-section configuration of CNT will be such that the stretching of the C-C bonds is minimal. On the other hand, the bending rigidity of graphene sheet is known to be rather small. In some cases, the elastic energy of bending can be concentrated to create a sharp ridge. This happens, for example, for $\mathrm{CNT}_{(55,55)}$, as can be seen in Figure 3(c). For CNTs with other chiralities the sharp ridges are not observed.

To further analyze the collapsed structures of CNTs, radial distribution functions (RDF), $g(r)$, are presented in Figure 4 for $\mathrm{CNT}_{(96,0)}$ (thin solid line), $\mathrm{CNT}_{(55,55)}$ (thick solid line), and $\mathrm{CNT}_{(50,60)}$ and $\mathrm{CNT}_{(40,70)}$ (dashed line). Note that the coordination shells for single-layer flat graphene have radii $\rho, \sqrt{3} \rho, 2 \rho, \sqrt{7} \rho \ldots$, where $\rho$ is the $\mathrm{C}-\mathrm{C}$ bond length. For $\rho=1.42 \AA$ the main peaks of RDF are expected at 1.42, 2.46, $2.84,3.75 \ldots \AA$, which is in good agreement with the results shown in Figure 4 . The small and relatively broad peak near 3.4 $\AA$, also shown in the inset of Figure 4, can be attributed to 


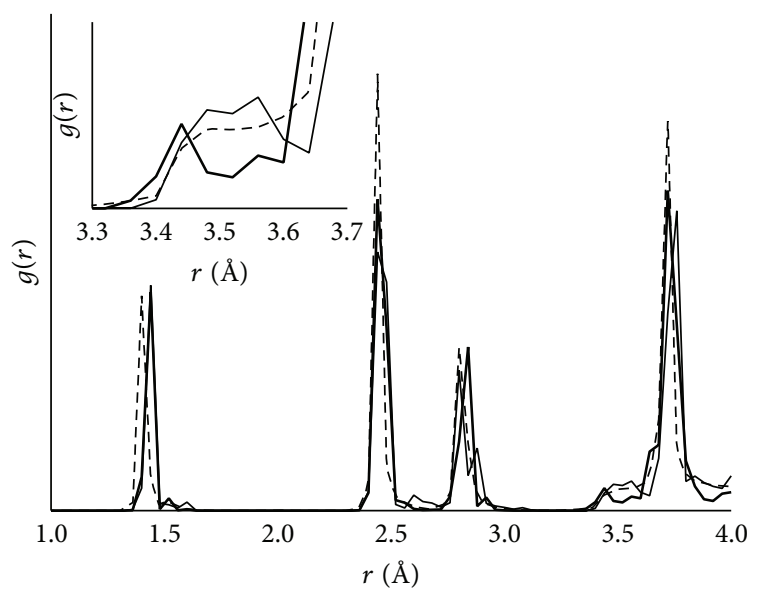

FIgURE 4: Radial distribution functions for $\mathrm{CNT}_{(96,0)}$ (thin solid line), $\mathrm{CNT}_{(55,55)}$ (thick solid line), and $\mathrm{CNT}_{(50,60)}$ and $\mathrm{CNT}_{(40,70)}$ (dashed line).

the van der Waals bonds between layers in bilayer graphene. It can be seen that for the zigzag and chiral CNTs this peak is broader than for the armchair nanotube, meaning that the coherency of layers in region I for the nanotubes of different chirality is different. For the nanotubes $\mathrm{CNT}_{(50,60)}$ and $\mathrm{CNT}_{(40,70)}$, RDF is almost the same, as shown by the dashed line in Figure 4.

\section{Conclusions}

The energy distribution and structure of collapsed carbon nanotubes of different diameter, length, and chirality have been investigated using molecular dynamics simulations. The obtained results revealed that the relaxed shape of the collapsed CNTs strongly depends on the chirality and other geometry parameters. For CNTs with different chiralities, we have observed different ripple configurations of the bilayer graphene region as well as different structures of the edges (see Figure 3). This is important because the ripples and cross-sectional profile can considerably change the mechanical and physical properties of CNTs.

The highest potential energy density in collapsed CNTs is observed along the ridges, which appear on the collapsed circled edges, while bilayer graphene has the lowest energy density. Taking into account the ability of collapsed CNT to return to the tubular metastable state through a reversible thermodynamic process explained in [10], it is possible to develop novel structures with anomalous thermal expansion and other anomalous properties in the temperature range where such transformation takes place. The results are therefore expected to provide new insights that will enhance the design of collapsed CNT-based devices and materials. The process of collapse and its effect on the properties as well as interplay between curvature energy and van der Waals energy may also be relevant to the collapse of larger graphitic structures such as carbon whiskers and are relevant to the description of the structure and properties of crumpled graphene $[41,42]$.

\section{Conflict of Interests}

The authors declare that there is no conflict of interests regarding the publication of this paper.

\section{Acknowledgments}

The authors acknowledge financial support by the National Natural Science Foundation of China (Grant no. 11472200) and the Open Fund of the Key Laboratory for Metallurgical Equipment and Control of Ministry of Education in Wuhan University of Science and Technology, China (Grant no. 2014C01). Julia A. Baimova and Sergey V. Dmitriev acknowledge financial support provided by the Russian Science Foundation through Grant no. 14-13-00982.

\section{References}

[1] S. Iijima, "Helical microtubules of graphitic carbon," Nature, vol. 354, no. 6348, pp. 56-58, 1991.

[2] O. Herranen, D. Talukdar, and M. Ahlskog, "Ultra-low noise multiwalled carbon nanotube transistors," Carbon, vol. 76, pp. 71-76, 2014.

[3] L. Wang and L. Cheng, "Piezoresistive effect of a carbon nanotube silicone-matrix composite," Carbon, vol. 71, pp. 319331, 2014.

[4] A. Mathur, S. S. Roy, K. S. Hazra, D. S. Misra, and J. A. McLaughlin, "Growth of carbon nanotube arrays using nanosphere lithography and their application in field emission devices," Diamond and Related Materials, vol. 19, no. 7-9, pp. 914-917, 2010.

[5] Q. Zhou, F. Meng, Z. Liu, and S. Shi, "The thermal conductivity of carbon nanotubes with defects and intramolecular junctions," Journal of Nanomaterials, vol. 2013, Article ID 842819, 7 pages, 2013.

[6] K.-S. Lin, Y.-J. Mai, S.-R. Li, C.-W. Shu, and C.-H. Wang, "Characterization and hydrogen storage of surface-modified multiwalled carbon nanotubes for fuel cell application," Journal of Nanomaterials, vol. 2012, Article ID 939683, 12 pages, 2012.

[7] G. Overney, W. Zhong, and D. Tománek, "Structural rigidity and low frequency vibrational modes of long carbon tubules," Physica D, vol. 27, no. 1, pp. 93-96, 1992.

[8] J. Tersoff and R. S. Ruoff, "Structural properties of a carbonnanotube crystal," Physical Review Letters, vol. 73, no. 5, pp. 676679, 1994.

[9] T. Chang, "Dominoes in carbon nanotubes," Physical Review Letters, vol. 101, no. 17, Article ID 175501, 4 pages, 2008.

[10] T. Chang and Z. Guo, "Temperature-induced reversible dominoes in carbon nanotubes," Nano Letters, vol. 10, no. 9, pp. 34903493, 2010.

[11] N. G. Chopra, L. X. Benedict, V. H. Crespi, M. L. Cohen, S. G. Louie, and A. Zettl, "Fully collapsed carbon nanotubes," Nature, vol. 377, no. 6545, pp. 135-138, 1995.

[12] L. X. Benedict, N. G. Chopra, M. L. Cohen, A. Zettl, S. G. Louie, and V. H. Crespi, "Microscopic determination of the interlayer binding energy in graphite," Chemical Physics Letters, vol. 286, no. 5-6, pp. 490-496, 1998.

[13] G. Gao, T. Çaǧin, and W. A. Goddard III, "Energetics, structure, mechanical and vibrational properties of single-walled carbon nanotubes," Nanotechnology, vol. 9, no. 3, pp. 184-191, 1998. 
[14] M.-F. Yu, M. J. Dyer, and R. S. Ruoff, "Structure and mechanical flexibility of carbon nanotube ribbons: an atomic-force microscopy study," Journal of Applied Physics, vol. 89, no. 8, pp. 4554-4557, 2001.

[15] B. Liu, M. F. Yu, and Y. Huang, "Role of lattice registry in the full collapse and twist formation of carbon nanotubes," Physical Review B-Condensed Matter and Materials Physics, vol. 70, no. 16, Article ID 161402, 2004.

[16] T. Tang, A. Jagota, C.-Y. Hui, and N. J. Glassmaker, "Collapse of single-walled carbon nanotubes," Journal of Applied Physics, vol. 97, no. 7, Article ID 074310, 2005.

[17] T. Chang, J. Hou, and X. Guo, "Reversible mechanical bistability of single-walled carbon nanotubes under axial strain," Applied Physics Letters, vol. 88, no. 21, Article ID 211906, 2006.

[18] R. Martel, T. Schmidt, H. R. Shea, T. Hertel, and Ph. Avouris, "Single- and multi-wall carbon nanotube field-effect transistors," Applied Physics Letters, vol. 73, no. 17, pp. 2447-2449, 1998.

[19] C. J. Park, Y. H. Kim, and K. J. Chang, "Band-gap modification by radial deformation in carbon nanotubes," Physical Review B-Condensed Matter and Materials Physics, vol. 60, Article ID 10656, 1999.

[20] J.-Q. Lu, J. Wu, W. Duan, F. Liu, B.-F. Zhu, and B.-L. Gu, "Metalto-semiconductor transition in squashed armchair carbon nanotubes," Physical Review Letters, vol. 90, Article ID 156601, 2003.

[21] J. Xiao, B. Liu, Y. Huang, J. Zuo, K.-C. Hwang, and M.-F. $\mathrm{Yu}$, "Collapse and stability of single- and multi-wall carbon nanotubes," Nanotechnology, vol. 18, Article ID 395703, 2007.

[22] B. Liu, Y. Huang, H. Jiang, S. Qu, and K. C. Hwang, "The atomicscale finite element method," Computer Methods in Applied Mechanics and Engineering, vol. 193, no. 17-20, pp. 1849-1864, 2004.

[23] M. F. Yu, M. J. Dyer, J. Chen, D. Qian, W. K. Liu, and R. S. Ruoff, "Locked twist in multiwalled carbon-nanotube ribbons," Physical Review B-Condensed Matter and Materials Physics, vol. 64, Article ID 241403(R), 2001.

[24] T. Tang and N. J. Glassmaker, "On the inextensible elastica model for the collapse of nanotubes," Mathematics and Mechanics of Solids, vol. 15, no. 5, pp. 591-606, 2010.

[25] K. V. Shanavas and S. M. Sharma, "Molecular dynamics simulations of phase transitions in argon-filled single-walled carbon nanotube bundles under high pressure," Physical Review B, vol. 79, no. 15, Article ID 155425, 2009.

[26] A. Liao, R. Alizadegan, Z.-Y. Ong et al., "Thermal dissipation and variability in electrical breakdown of carbon nanotube devices," Physical Review B, vol. 82, Article ID 205406, 2010.

[27] X. Yang and G. Wu, "The study of structural, electronic and optical properties of double-walled carbon nanotube bundles under hydrostatic pressure," Europhysics Letters, vol. 81, no. 4, Article ID 47003, 2008.

[28] J. Sandler, M. S. P. Shaffer, A. H. Windle et al., "Variations in the Raman peak shift as a function of hydrostatic pressure for various carbon nanostructures: a simple geometric effect," Physical Review B, vol. 67, Article ID 035417, 2003.

[29] J. Tang, L.-C. Qin, T. Sasaki, M. Yudasaka, A. Matsushita, and S. Iijima, "Compressibility and polygonization of single-walled carbon nanotubes under hydrostatic pressure," Physical Review Letters, vol. 85, no. 9, Article ID 1887, 2000.

[30] S. Karmakar, S. M. Sharma, P. V. Teredesai et al., "Structural changes in single-walled carbon nanotubes under nonhydrostatic pressures: X-ray and Raman studies," New Journal of Physics, vol. 5, article 143, 2003.
[31] J. A. Baimova, B. Liu, S. V. Dmitriev, and K. Zhou, "Mechanical properties and structures of bulk nanomaterials based on carbon nanopolymorphs," Physica Status Solidi-Rapid Research Letters, vol. 8, no. 4, pp. 336-340, 2014.

[32] J. A. Baimova, B. Liu, S. V. Dmitriev, N. Srikanth, and K. Zhou, "Mechanical properties of bulk carbon nanostructures: effect of loading and temperature," Physical Chemistry Chemical Physics, vol. 16, no. 36, pp. 19505-19513, 2014.

[33] S. Plimpton, "Fast parallel algorithms for short-range molecular dynamics," Journal of Computational Physics, vol. 117, no. 1, pp. 1-19, 1995.

[34] S. J. Stuart, A. B. Tutein, and J. A. Harrison, "A reactive potential for hydrocarbons with intermolecular interactions," The Journal of Chemical Physics, vol. 112, no. 14, pp. 6472-6486, 2000.

[35] B. Liu, C. D. Reddy, J. Jiang et al., "Morphology and inplane thermal conductivity of hybrid graphene sheets," Applied Physics Letters, vol. 101, no. 21, Article ID 211909, 2012.

[36] B. Liu, C. D. Reddy, J. Jiang et al., "Thermal conductivity of silicene nanosheets and the effect of isotopic doping," Journal of Physics D: Applied Physics, vol. 47, no. 16, Article ID 165301, 2014.

[37] B. Liu, J. A. Baimova, S. V. Dmitriev, X. Wang, H. Zhu, and K. Zhou, "Discrete breathers in hydrogenated graphene," Journal of Physics D: Applied Physics, vol. 46, no. 30, Article ID 305302, 2013.

[38] J. A. Baimova, B. Liu, and K. Zhou, "Folding and crumpling of graphene under biaxial compression," Letters on Materials, vol. 4, no. 2, pp. 96-99, 2014.

[39] J. A. Baimova, S. V. Dmitriev, K. Zhou, and A. V. Savin, "Unidirectional ripples in strained graphene nanoribbons with clamped edges at zero and finite temperatures," Physical Review B: Condensed Matter and Materials Physics, vol. 86, no. 3, Article ID 035427, 2012

[40] J. A. Baimova, S. V. Dmitriev, and K. Zhou, "Strain-induced ripples in graphene nanoribbons with clamped edges," Physica Status Solidi B, vol. 249, no. 7, pp. 1393-1398, 2012.

[41] E. A. Korznikova, J. A. Baimova, S. V. Dmitriev, A. V. Korznikov, and R. R. Mulyukov, "Mechanical behavior of crumpled sheet materials subjected to uniaxial compression," Reviews on Advanced Materials Science, vol. 39, pp. 92-98, 2014.

[42] J. A. Baimova, E. A. Korznikova, S. V. Dmitriev, B. Liu, and K. Zhou, "Review on crumpled graphene: unique mechanical properties," Reviews on Advanced Materials Science, vol. 39, pp. 69-83, 2014. 

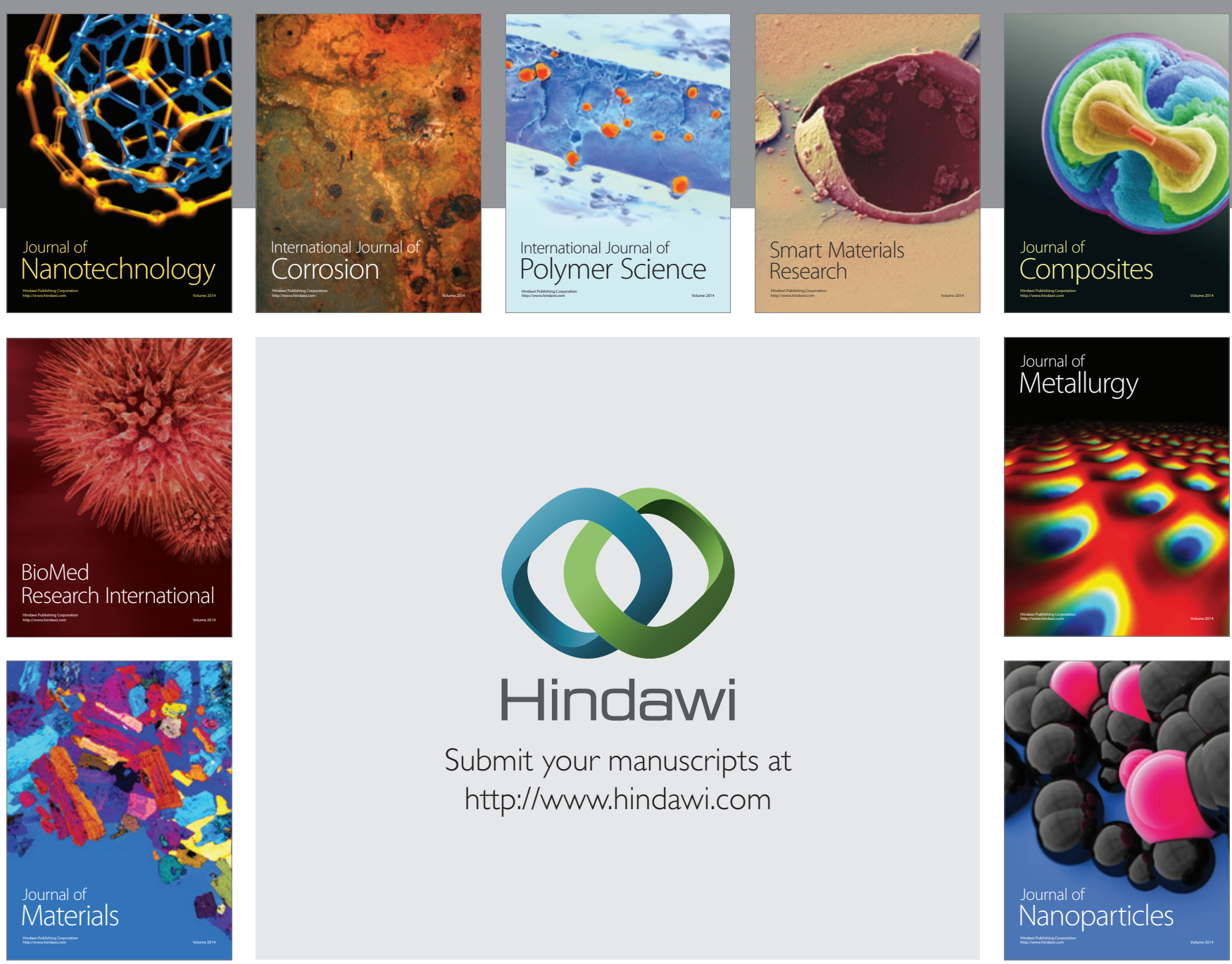

Submit your manuscripts at http://www.hindawi.com
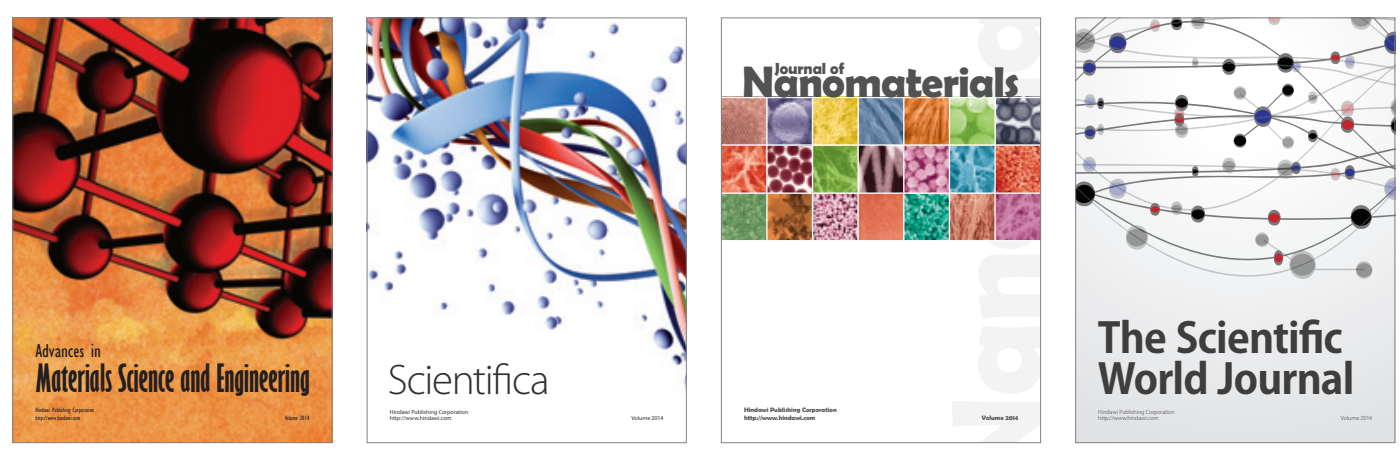

\section{The Scientific World Journal}
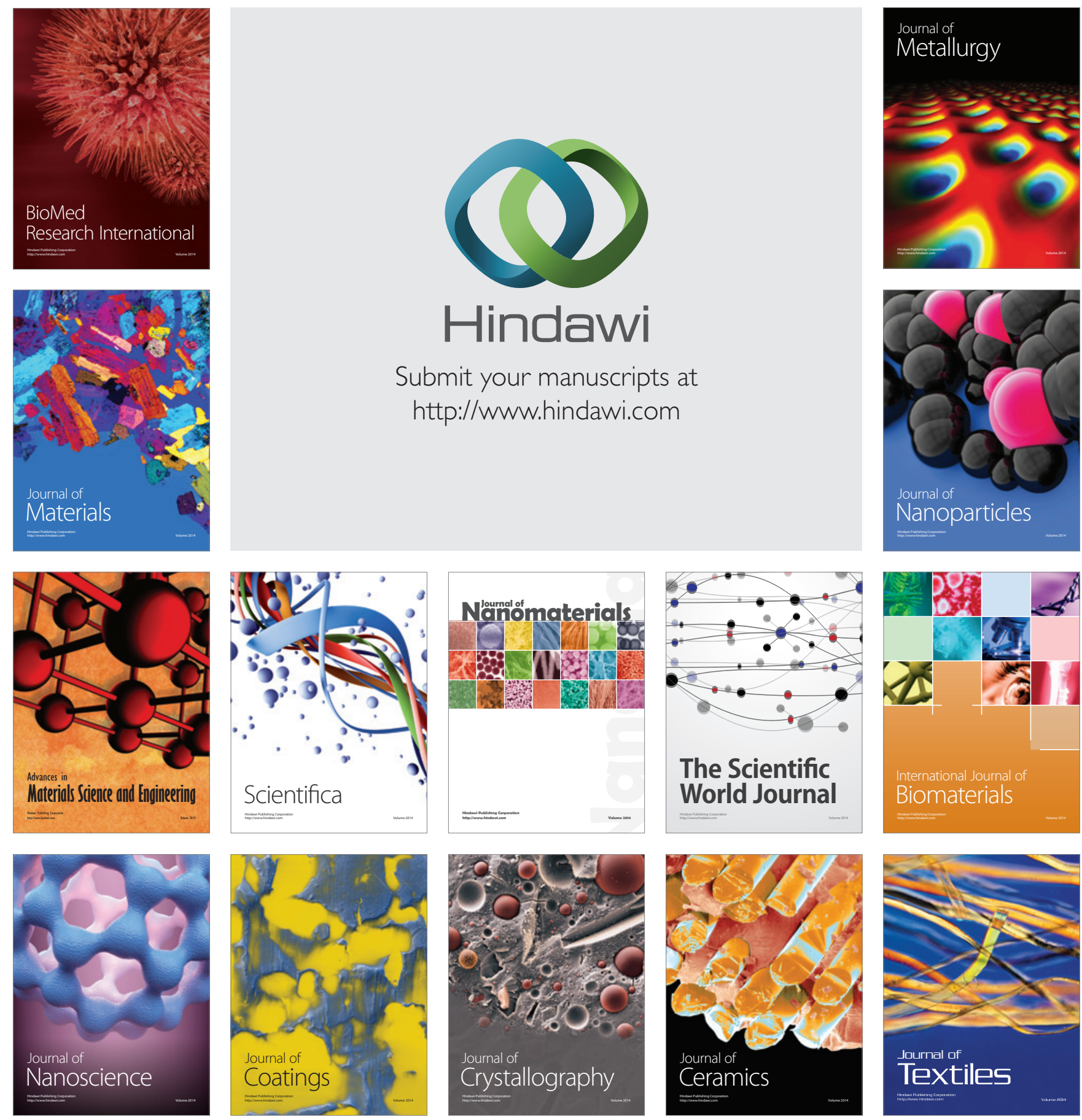